\begin{tabular}{c} 
International Journal of Engineering \& Technology, 7 (4.36) (2018) 750-752 \\
International Journal of Engineering \& Technology \\
SPC \\
Website: www.sciencepubco.com/index.php/IJET \\
Research paper \\
\hline
\end{tabular}

\title{
Prime Graceful Labeling
}

\author{
T.M. Selvarajan ${ }^{1 *}$, R. Subramoniam ${ }^{2}$ \\ ${ }^{1}$ Department of Mathematics, Noorul Islam University, Kanyakumari, India. \\ ${ }^{2}$ Department of Mathematics, Lekshmipuram College of Arts and Science, Neyyoor, Tamilnadu, India.
}

\begin{abstract}
A graph $\mathrm{G}$ with $\mathrm{m}$ vertices and $\mathrm{n}$ edges, is said to be prime graceful labeling, if there is an injection $\varphi$ from the vertices of $\mathrm{G}$ to $\{1,2, \ldots$, $\mathrm{k}\}$ where $\mathrm{k}=\min \{2 \mathrm{~m}, 2 \mathrm{n}\}$ such that $\operatorname{gcd}\left(\varphi\left(\mathrm{v}_{\mathrm{i}}\right), \varphi\left(\mathrm{v}_{\mathrm{j}}\right)\right)=1$ and the induced injective function $\varphi^{*}$ from the edges of $\mathrm{G}$ to $\{1,2, \ldots, \mathrm{k}-$ $1\}$ defined by $\varphi^{*}\left(\mathrm{v}_{\mathrm{i}} \mathrm{v}_{\mathrm{j}}\right)=\left|\varphi\left(\mathrm{v}_{\mathrm{i}}\right)-\varphi\left(\mathrm{v}_{\mathrm{j}}\right)\right|$, the resulting edge labels are distinct. In this paper path $P_{n}$, cycle $\mathrm{C}_{\mathrm{n}}$, star $\mathrm{K}_{1, \mathrm{n}}$, friendship graph $F_{n}$, bistar $B_{n, n}, C_{4} \cup P_{n}, K_{m, 2}$ and $K_{m, 2} \cup P_{n}$ are shown to be Prime Graceful Labeling .
\end{abstract}

Keywords: Prime labeling, graceful labeling and prime graceful labeling.

\section{Introduction}

By a graph $\mathrm{G}=(\mathrm{V}, \mathrm{E})$, we mean a finite simple undirected graph. For standard terminology and notations related to graph theory we refer F. Harary [2] and J.A.Bondy and U.S.R.Murthy [3]. For various graph labeling problems, we refer to Gallian [4]. We provide here some definitions which are necessary for our present investigations. In this paper, the concept of Prime Graceful labeling is introduced and some results on these are established.

\section{Definition 1.1}

Let $G=((V)(G),(E(G))$ be a graph with $m$ vertices. A bijection $f$ : $\mathrm{V} \rightarrow\{1,2, \ldots, \mathrm{m}\}$ is called a prime labeling if for each edge $\mathrm{e}=\mathrm{uv}$, $\operatorname{gcd}(\mathrm{f}(\mathrm{u}), \mathrm{f}(\mathrm{v}))=1$. A graph which admits a prime labeling is called a Prime graph.

\section{Definition 1.2}

Let $\mathrm{G}=((\mathrm{V}(\mathrm{G}),(\mathrm{E}(\mathrm{G}))$ be a simple, finite and undirected graph with $|\mathrm{V}|=\mathrm{m}$ and $|\mathrm{E}|=\mathrm{n}$. An injective function $\mathrm{f}: \mathrm{V} \rightarrow\{1, \overline{2}, \ldots$, $\mathrm{m}$ \} is called a graceful labeling of $\mathrm{G}$ if all the edge labels of $\mathrm{G}$ given by $f(u v)=|f(u)-f(v)|$ for every $u v \in E$ are distinct. $A$ graph which admits a graceful labeling is called a graceful graph.

\section{Definition 1.3}

A graph $\mathrm{G}$ with $\mathrm{m}$ vertices and $\mathrm{n}$ edges, is said to be prime graceful labeling, if there is an injection $\varphi$ from the vertices of $\mathrm{G}$ to $\{1,2, \ldots, \mathrm{k}\}$ where $\mathrm{k}=\min \{2 \mathrm{~m}, 2 \mathrm{n}\}$ such that $\operatorname{gcd}\left(\varphi\left(\mathrm{v}_{1}\right)\right.$, $\left.\varphi\left(\mathrm{v}_{1}\right)\right)=1$ and the induced injective function $\varphi^{*}$ from the edges of $\mathrm{G}$ to $\{1,2, \ldots, \mathrm{k}-1\}$ defined by $\varphi^{*}\left(\mathrm{v}_{1} \mathrm{v}_{\mathrm{j}}\right)=\left|\varphi\left(\mathrm{v}_{1}\right)-\varphi\left(\mathrm{v}_{\mathrm{j}}\right)\right|$, the resulting edge labels are distinct.

\section{Example 1.4}

Triangular snake T2 is a prime graceful labeling

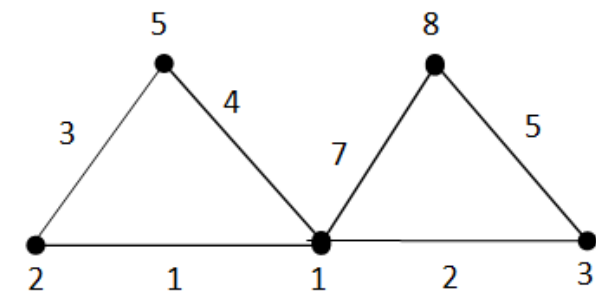

Example 1.5

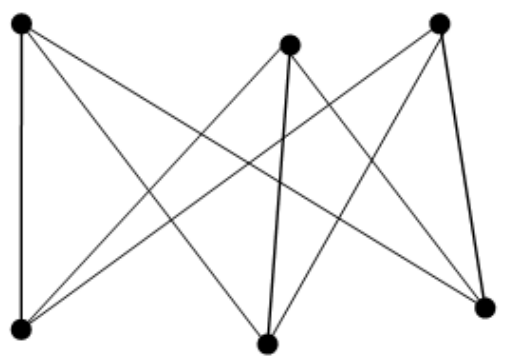

In $\mathrm{k} 3,3$, edges and vertices cannot be labeled, so that GCD of end vertices of each edge is one and edge labels are distinct. So k3, 3 is not a prime graceful labeling.

\section{Theorem 1.6}

The star graph $\mathrm{k} 1, \mathrm{n}$ admits prime graceful labeling.

Proof

The star graph $\mathrm{k} 1, \mathrm{n}$ has $\mathrm{n}+1$ vertices and $\mathrm{n}$ edges.

$$
\begin{aligned}
\mathrm{k}=\min & \{2(\mathrm{n}+1), 2 \mathrm{n}\} \\
& =2 \mathrm{n}
\end{aligned}
$$

In $\mathrm{k} 1, \mathrm{n}$, one vertex is adjacent with remaining $\mathrm{n}$ vertices.

Label the vertex of degree $n$ with one remaining with $2,3,4, \ldots$, $\mathrm{n}, \mathrm{n}+1$.

The GCD of end vertices of each edge is 1

The edges labels $1,2,3,4, \ldots$ are distinct. 


\section{Example 1.7}

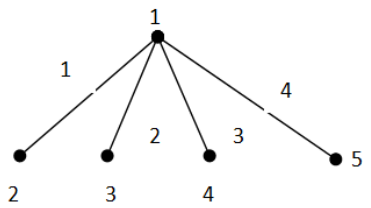

\section{Theorem 1.8}

The Bistar graph $\mathrm{Bn}, \mathrm{n}$ admits prime graceful labeling.

\section{Proof}

The Bistar graph has $(2 n+2)$ vertices and $(2 n+1)$ edges.

Bistar graph $\mathrm{Bn}, \mathrm{n}$ has exactly two vertices of degree $\mathrm{n}$, label these vertices by 1 and 3 .

$$
\begin{gathered}
\mathrm{k}=\min \{2(2 \mathrm{n}+2), 2(2 \mathrm{n}+1)\} \\
=2(2 \mathrm{n}+1)
\end{gathered}
$$

Label the vertices that are adjacent with vertex label 1 by $2,4,5$, $6, \ldots, n+2$, so that gcd of end vertices of each edge is one.

Label the vertices that are adjacent with vertex label 3 from the set $\{\mathrm{n}+3, \mathrm{n}+4, \ldots, 2(2 \mathrm{n}+1)\}$ and not a multiple of 3 .

The gcd of end vertices of each edge is 1 and edge labels are distinct.

\section{Example 1.9}

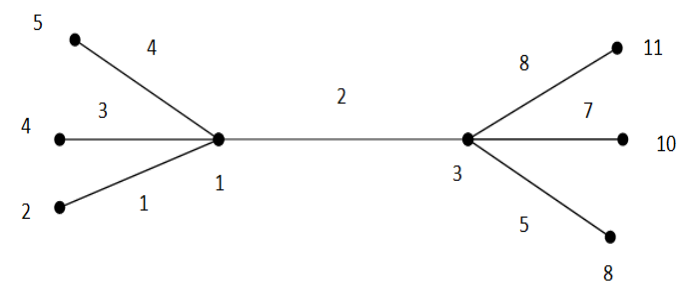

\section{Theorem 1.10}

The path Pn admits prime graceful labeling.

\section{Proof}

The path Pn has $\mathrm{n}$ and vertices $(\mathrm{n}-1)$ edges.

$$
\begin{gathered}
\mathrm{k}=\min \{2 \mathrm{n}, 2(\mathrm{n}-1)\} \\
=2 \mathrm{n}-2
\end{gathered}
$$

The vertices of $P n$ are labeled from the set $S=\{1,2,3, \ldots, 2 n-3$, $2 n-2\}$

Choose $\left(\frac{n}{2}\right)^{\text {th }}$ vertex and label it with 1 .

If $\mathrm{n}$ is odd, choose $\left(\frac{n+1}{2}\right)^{\text {th }}$ vertex and label it with 1 .

Choose last two integers from the set $\mathrm{S}$.

i.e., $(2 n-3)$ and $(2 n-2)$ and label it to the adjacent vertices of vertex label 1 .

Choose two integers from the beginning of the set $\mathrm{S}$ and label with the vertex adjacent to the vertex label $2 n-3$ and $2 n-2$, so that the gcd of two consecutive vertices is 1 . Alternatively choose the integers from the beginning the set and end of the set, label the vertices so that gcd of two consecutive vertices is 1 . The resulting edge labels are distinct.

\section{Example 1.11}

$\mathrm{P}_{8}$ is prime graceful labeling.

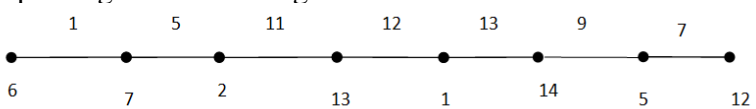

\section{Theorem 1.12}

The friendship graph $\mathrm{F}_{\mathrm{n}}$ admits prime graceful labeling.

\section{Proof}

The friendship graph $F_{n}$ has $(2 n+1)$ vertices and $3 n$ edges.

$$
\begin{gathered}
\mathrm{k}=\min \{2(2 n+1), 6 n\} \\
=\min \{4 n+2,6 n\} \\
=4 n+2
\end{gathered}
$$

In friendship graph, one vertex of degree $2 \mathrm{n}$ is adjacent to the remaining $2 \mathrm{n}$ vertices, label the vertex of degree $2 \mathrm{n}$ with 1 . Choose a vertex from each cycle $\mathrm{C}_{3}$, label it with $2,3,4,5, \ldots,(\mathrm{n}+$ $1)$ and label the remaining vertices with $(4 n+2),(4 n+1),(4 n)$, $(4 n-1) \ldots$, so that the gcd of end vertices of each edge is 1 .

The resulting edge labels are distinct.

\section{Example 1.13}

Friendship graph $\mathrm{F}_{4}$ is prime graceful labeling.

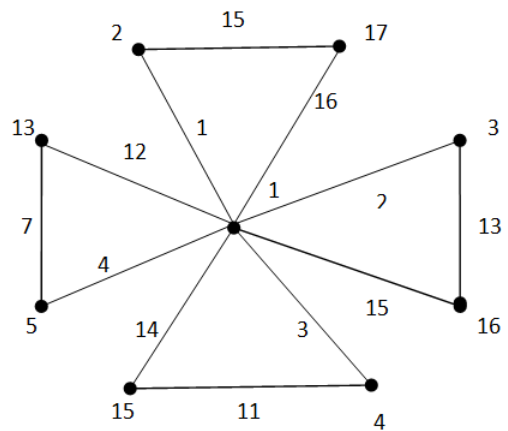

\section{Theorem 1.14}

The cycle graph $\mathrm{Cn}$ admits prime graceful labeling.

\section{Proof}

The cycle $C_{n}$ has $n$ edges and $n$ vertices.

$$
\begin{gathered}
K=\min (2 n, 2 n) \\
=2 n .
\end{gathered}
$$

The vertices of $C_{n}$ are labeled from the set $S=\{1,2,3, \ldots, 2 n-1$, 2n\}.

Choose an arbitrary vertex in $\mathrm{C}_{\mathrm{n}}$ and label it with 1 .

Choose last two integers from the set $\mathrm{S}$.

i.e., $2 n-1$ and $2 n$ label it to the adjacent vertices of vertex label 1 .

Choose the integers from the beginning of the set $\mathrm{S}$ and label with the vertex adjacent to the vertex label $2 n-1$ or $2 n$, so that the gcd of two consecutive vertices is 1 and edge labels are distinct.

\section{Example 1.15}

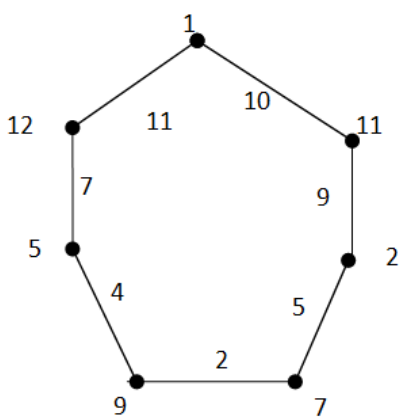

\section{Theorem 1.16}

The graph $K_{m, 2}$ admits prime graceful labeling for $m \geq 2$. 


\section{Proof}

The graph $K_{m, 2}$ contains $(m+2)$ vertices and $2 m$ edges.

$$
\begin{gathered}
\mathrm{K}=\min \{2(\mathrm{~m}+2), 2(2 \mathrm{~m})\} \\
=\min \{2 \mathrm{~m}+4,4 \mathrm{~m}\} \\
=2 \mathrm{~m}+4 .
\end{gathered}
$$

Label the vertices having degree $m$ of $K_{m, 2}$ with 1 and 2 remaining vertices with odd numbers $3,5, \ldots,[(2 m+4)-1]$. Hence the gcd of two consecutive vertices of each edge is 1 and resulting edge labels are distinct.

\section{Example 1.17}

The graph $\mathrm{K}_{5,2}$ is prime graceful labeling.

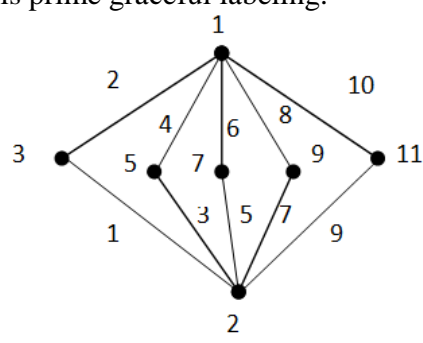

\section{Theorem 1.18}

The Comb graph Pn $\odot \mathrm{K} 1$ admits prime graceful labeling.

\section{Proof}

The Comb graph Pn $\odot \mathrm{K} 1$ has $2 \mathrm{n}$ vertices and $(2 \mathrm{n}-1)$ edges.

$$
\begin{gathered}
\mathrm{k}=\min \{4 \mathrm{n}, 4(\mathrm{n}-1)\} \\
=4(2 \mathrm{n}-1) \\
=8 \mathrm{n}-4
\end{gathered}
$$

The vertices of $P n$ are labeled from the set $S=\{1,2,3, \ldots, 8 n-$ $4\}$ as in Theorem1.10. Label the remaining $n$ vertices, so that gcd of two consecutive vertices of each edge is 1 and resulting edge labels are distinct.

\section{Example 1.19}

$\mathrm{P} 4 \odot \mathrm{K} 1$ is prime graceful labeling.

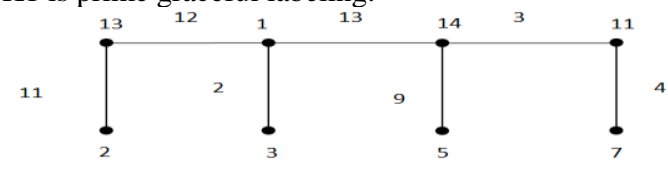

\section{Theorem 1.20}

The graph Pn $\cup$ C4 admits prime graceful labeling.

\section{Proof}

The graph Pn $\cup C 4$ contains $(n+4)$ vertices and $(n+3)$ edges.

$$
\begin{gathered}
K=\min \{2(n+4), 2(n+3)\} \\
=\min \{2 n+8,2 n+6\} \\
=2 n+6
\end{gathered}
$$

Label the vertices of Pn with $v_{1}, v_{2}, \ldots, v_{n}$, the vertices are labeled from the set $S=\{1,2, \ldots, 2 n+6\}$ as in Theorem1.10 .

Label the vertices of $C 4$ from the set $\{2 n, 2 n+1, \ldots, 2 n+6\}$, so that the gcd of two consecutive vertices is 1 and the edge labels are distinct.

\section{Example 1.21}

The graph C4 $\cup$ P5 is prime graceful labeling.
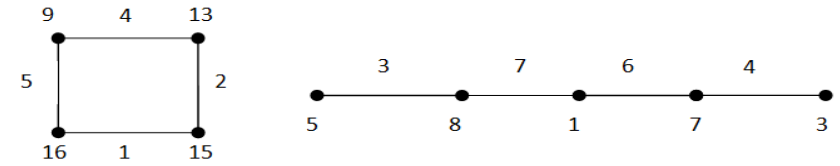

\section{Theorem 1.22}

The graph $\mathrm{Pn} \cup \mathrm{Km}, 2(\mathrm{~m} \geq 1)$ admits prime graceful labeling.

\section{Proof}

The graph $K m, 2 \cup$ Pn contains $(m+n+2)$ vertices and $(2 m+n-$ 1) edges.

$$
\begin{gathered}
\mathrm{K}=\min \{2(\mathrm{~m}+\mathrm{n}+2), 2(2 \mathrm{~m}+\mathrm{n}-1)\} \\
=\min \{2 \mathrm{~m}+2 \mathrm{n}+4,4 \mathrm{~m}+2 \mathrm{n}-2\} \\
=2 \mathrm{~m}+2 \mathrm{n}+4 .
\end{gathered}
$$

Label the vertices of Pn with v1, v2, ..., vn , the vertices are labeled from the set

$\mathrm{S}=\{1,2, \ldots, 2 \mathrm{~m}+2 \mathrm{n}+4\}$ as in Theorem1.10 . Label the vertices having degree

$2 \mathrm{~m}$ of $\mathrm{Km}, 2$ with 1 and 2 and remaining vertices with odd numbers $3,5,7, \ldots$.

Hence the gcd of two adjacent vertices is 1 and edge labels are distinct.

\section{Example 1.23}

The graph K3,2 $\cup \mathrm{P} 5$ is prime graceful labeling.

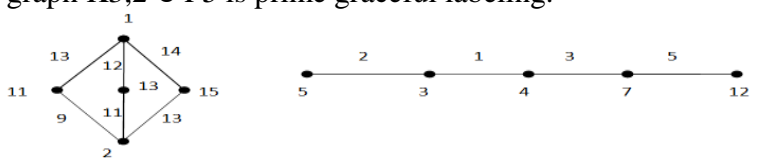

\section{Conclusion}

In this paper the concept of prime graceful labeling is introduced and prove the existence of prime graceful labeling for graphs such as path $P n$, cycle $C_{n}$, star $K_{1, n}$, friendship graph $F_{n}$, bistar $B_{n, n}, C_{4}$ $\cup P_{n}, K_{m, 2}$ and $K_{m, 2} \cup P_{n}$.

It would be interesting to do further research on this topic and to find more graphs that satisfy the prime graceful labeling.

\section{References}

[1] Tout R, Dabboucy AN \& Howalla K, "Prime labeling of graphs", National Academy Science Letters-India, Vol.5, No.11, (1982) pp.365-368.

[2] Harary F, Graph Theory, Addison-Wesley, Reading, Mass, (1969).

[3] Bondy JA \& Murthy USR, "Graph theory with applications", Elsevier North Holland. New York, (1976).

[4] Gallian JA, "A dynamic survey of graph labeling", The electronic journal of combinatory, Vol.16, No.6, (2009), pp.1-219.

[5] Rosa A, "On certain valuations of the vertices of a graph", Theory of Graphs (Internet Symposium, Rome, (1966), pp.349-355.

[6] Uma R \& Murugesan, N, "Graceful labeling of some graphs and their sub graphs", Asian Journal of Current Engineering and Maths, Vol.1, No.6, (2013), pp.307-370.

[7] Vaidya SK \& Vihol PL, "Prime cordial labeling for some cycle related graphs", International Journal of Open Problems in Computer Science and Mathematics, Vol.3, No.5, (2010), pp.98104

[8] Vaidya SK \& Shah NH, "Graceful and odd graceful labeling of some graphs", International journal of Mathematics and soft computing, Vol.3, No.1, (2013), pp.61-68.

[9] Singhun S, "Graphs with line-odd Graceful labeling", International Mathematics Forum, Vol.8, No.12, (2010), pp.577-582.

[10] Sridevi R, Navaneethakrishnan S \& Nagarajan K, "Super Fibonacci graceful labeling of some special class of graphs", Mathematical Combinatory, Vol.1, (2011), pp.59-72. 